\title{
Comparison between optical techniques and confocal microscopy for defect detection on thin wires
}

\author{
Philip Siegmann ${ }^{\mathrm{a},{ }^{*}}$, Luis Miguel Sanchez-Brea ${ }^{\mathrm{b}}$, Juan Carlos Martinez-Anton ${ }^{\mathrm{b}}$, \\ Eusebio Bernabeu ${ }^{\mathrm{a}}$ \\ ${ }^{a}$ Departamento de Óptica, Universidad Complutense de Madrid, Facultad de Ciencias Físicas, \\ Ciudad Universitaria s.n., 28040 Madrid, Spain \\ ${ }^{\mathrm{b}}$ Departamento de Óptica, Universidad Complutense de Madrid, Escuela Universitaria de Óptica, \\ Av. Arcos del Jalón s.n., 20837 Madrid, Spain
}

Available online 11 September 2004

\begin{abstract}
Conventional microscopy techniques, such as atomic force microscopy (AFM), scanning electron microscopy (SEM), and confocal microscopy (CM) are not suitable for on-line surface inspection of fine metallic wires. In the recent years, some optical techniques have been developed to be used for those tasks. However, they need a rigorous validation. In this work, we have used confocal microscopy to obtain the topography $\mathrm{z}(\mathrm{x}, \mathrm{y})$ of wires with longitudinal defects, such as dielines. The topography has been used to predict the light scattered by the wire. These simulations have been compared with experimental results, showing a good agreement.
\end{abstract}

(C) 2004 Published by Elsevier B.V.

PACS: 68.37.d; 68.37.Hk; 68.37.Ps; 81.20.Hy

Keywords: Wires; Surface structures; Surface defects; Metallic surfaces

\section{Introduction}

Thin metallic wires (50-1000 $\mu \mathrm{m}$ of diameter) are produced for a great number of applications. The quality of the surface of such wires plays an important role in the applications, since it may change some of their physical-chemical properties. The surface quality and the geometrical and dimensional accuracy of fine wires are decisively influenced by the stress, deformation, and temperature conditions, as well as

\footnotetext{
* Corresponding author. Present address: Department of Mechanical Engineering, The University of Sheffield, Mappin Street, Sheffield S1 3JD, UK.
}

the kinetic and tribological conditions occurring at the die/wire interface during wire drawing. In this regard, friction plays a critical role in the deformation process, since it improves smoothness of the surface structure. Defective pre-material or disruptions in the sensitive tribological/cold work system are the causes for obtaining unsatisfactory surface quality in ultra-fine wires.

Nevertheless, structures on the wire surface appear and they have been catalogued for thin metallic wires [1]. The surface structures found have been sorted into categories (laps, local structures, continuous structures, shape deviations, and damages) according to their shape and origin. 
Several conventional techniques are commonly used for surface characterization, such as scanning electron microscopy (SEM), atomic force microscopy (AFM), and confocal microscopy (CM). However, these techniques can only be used for off-line inspection, and just a small sample of the wire reel can be analyzed.

SEM is very suitable for thin wire surface characterization [2]. It presents very good resolution and depth of field. It presents a wide explored surface range in comparison to the wire diameters considered $(<600 \mu \mathrm{m})$. SEM, however, also presents several disadvantages: it is not possible to obtain topographical measurements for the determination of depths, and the scattered electrons are influenced by not only the slope but also the topography's curvature and, as a consequence, the edges are enhanced.

AFM has also been used for surface characterization, and it is a suitable technique for the characterization of plane [3] and cylindrical surfaces [4]. CM is another method to complement SEM. Both AFM and $\mathrm{CM}$ allow us to obtain three-dimensional topologies of wires. The advantage of CM with respect to AFM is the speed in obtaining images and the little sample preparation required.

All these methods of surface quality inspection for fine wires are not sufficient where demanding applications are involved, such as in production line inspection where the objective of quality control is to determine the surface quality of all the reel in real time. For these reasons, new techniques of defect detection have been developed especially devoted to cylindrical objects. Amongst them, optical methods have been proven of great interest [5].

Validation of such optical techniques has normally been made by comparing the results visually, with images of wires obtained by optical microscopy or SEM [5]. This is not the best way of comparing, since the real topography of wire is not taken into account.

In this work, we analyze briefly an optical method for cylinder surface inspection. The incident field is modeled as bundle of rays, each characterized by its amplitude, direction, and optical path. We assume that such rays propagate in straight lines and are reflected especularly by the wire surface. The far field amplitude is determined by summing all rays that have the same output direction. Experimental measurements are obtained with the optical technique and they are compared with the results predicted by the model. The results obtained are quite similar.

\section{Optical technique for surface structure detection}

In this section, we will briefly explain the optical technique of surface wire inspection. A laser beam falls obliquely on the wire surface and it is reflected by it forming a cone of light [6]. The cone of light transports information of the corresponding $180^{\circ}$ illuminated wire surface. Therefore, to analyze the entire wire surface at least three laser beams are required, shifted $120^{\circ}$ to each other. These three beams are necessary because of diffraction effects at the edge that produce dead angles. The scattered light, forming a ring, is collected by an optical lens system that sends light to a CCD camera [7].

\subsection{Model for scattering of wire with defects}

To analyze how light is scattered in terms of the wire topography, we use the geometrical approach. We considered that the light beam is formed by a bundle of rays propagating in straight lines. When the ray strikes on the wire surface, it is reflected with an angle equal to the incident one (Snell's law) [8]. In addition to its direction, other properties are associated to each ray: amplitude which for simplicity we will suppose to be unity, and phase, which is proportional to the optical path $\Delta$. Therefore, the amplitude of a point along its trajectory is $u=A$ $\exp (2 \pi \mathrm{i} \Delta / \lambda)$, where $A$ is the amplitude and $\lambda$ is the wavelength.

Let us assume that $N_{0}$ parallel rays, with a direction $\boldsymbol{k}_{0}=k(0,-1)$ and different locations $x_{\mathrm{R}}$, fall on the wire surface, characterized by its profile $f(x)$. The impact of such rays on the wire surface is at $P_{\mathrm{R}}=\left[x_{\mathrm{R}}\right.$, , $\left.y_{R}=f\left(x_{R}\right)\right]$. By simple geometrical considerations, it is obtained that the perpendicular vector to such points, $\boldsymbol{n}_{\mathrm{R}}$, is

$\boldsymbol{n}_{\mathrm{R}}=\frac{\left(-f^{\prime}, 1\right)}{\sqrt{f^{\prime 2}+1}}$,

the direction of the reflected rays $\boldsymbol{k}_{\mathrm{R}}$ is

$\boldsymbol{k}_{\mathrm{R}}=-2\left(\boldsymbol{k}_{0} \boldsymbol{n}_{\mathrm{R}}\right) \boldsymbol{n}_{\mathrm{R}}+\boldsymbol{k}_{0}$, 


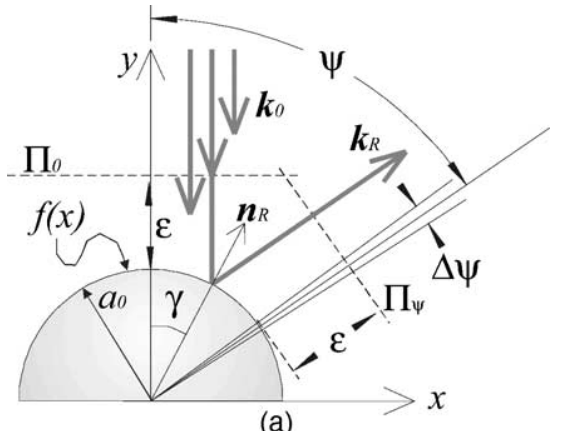

(a)

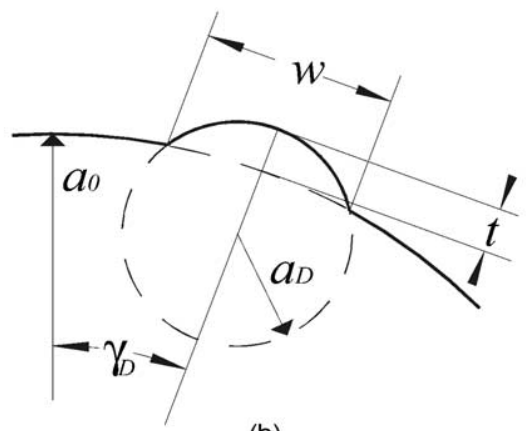

(b)

Fig. 1. (a) Scheme of how rays fall on the wire surface and parameters involved. (b) Definition of parameters of defect.

and the optical path $\Delta$ from planes $\Pi_{0}$ to $\Pi_{\psi}$ is (see Fig. 1a)

$\Delta_{\mathrm{R}}=2\left(a_{0}+\varepsilon\right)-x_{\mathrm{R}} \sin \psi_{\mathrm{R}}-y_{\mathrm{R}}\left(1+\cos \psi_{\mathrm{R}}\right)$,

where $\varepsilon$ is the distance between $\Pi_{0}$ and $\Pi_{\psi}$ planes, and $\psi_{\mathrm{R}}$ is the angle of scattering for the reflected ray. Those rays reflected with an angular range $(\psi-\Delta \psi / 2$, $\psi+\Delta \psi / 2)$ interfere. The output rays are collected by a CCD camera. The optical field, $u$, at each angular interval corresponding to each pixel is given by

$u(\psi ; \Delta \psi)=N_{\Delta \psi} \sum_{n=1}^{N_{\Delta \psi}} \exp \left(i k \Delta_{n}\right)$,

where $\Delta \psi$ is angular footprint of the corresponding pixel of the CCD camera, $N_{\Delta \psi}$ is the number of reflected rays towards the pixel and $\Delta_{n}$ is the optical path of such rays. The intensity obtained at each pixel is given by

$I(\psi ; \Delta \psi)=u(\psi ; \Delta \psi) u^{*}(\psi ; \Delta \psi)$.

When an experimental profile $f(x)$ is introduced in equations, the intensity pattern fluctuates strongly due to the short-range variations in the topography. There- fore, a better approach is to fit the topography of the defects to a slowly varying function. We have found that a profile that works well is that defined by

$$
f(x)=\left\{\begin{array}{c}
y_{\mathrm{D}}+a_{\mathrm{D}} \sqrt{1-\left(\frac{x-x_{\mathrm{D}}}{a_{\mathrm{D}}}\right)^{2}}, \\
x \in\left(x_{\mathrm{i}}, x_{\mathrm{s}}\right) \\
a_{0} \sqrt{1-\left(\frac{x}{a_{\mathrm{D}}}\right)^{2}}, \\
x \in\left(-a_{0}, x_{\mathrm{i}}\right) \cup\left(x_{\mathrm{s}}, a_{0}\right)
\end{array}\right.
$$

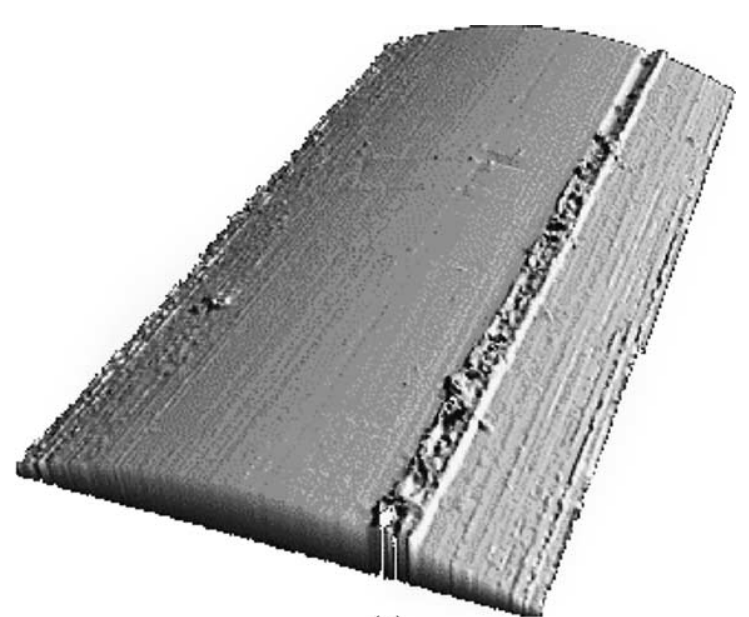

(a)

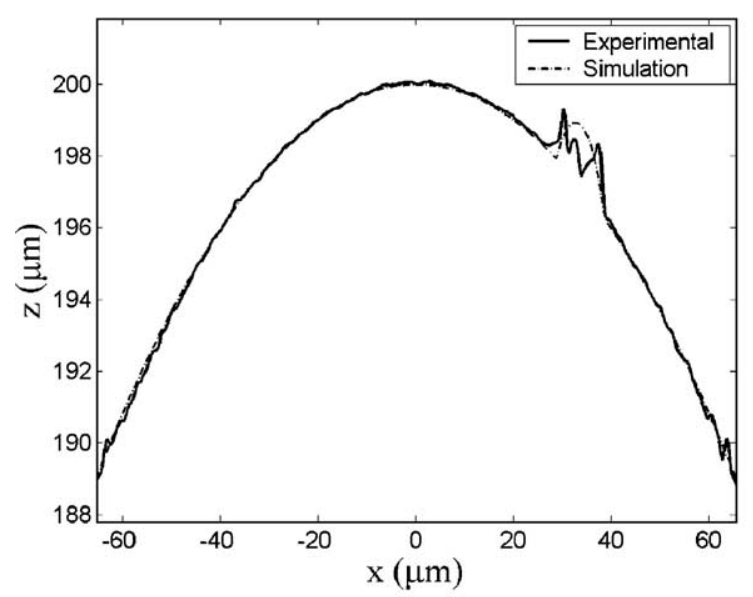

(b)

Fig. 2. (a) Topographic image of a wire surface obtained by confocal microscopy. The wire is made of steel and its radius is $200 \mu \mathrm{m}$. (b) Transversal profile (solid) and fit according to Eq. (6) (dashed) for the said wire. The parameters obtained are $a_{0}=$ $200 \mu \mathrm{m}, a_{\mathrm{D}}=8 \mu \mathrm{m}, w=10 \mu \mathrm{m}(t=1.67 \mu \mathrm{m})$. 
where $a_{0}$ and $a_{\mathrm{D}}$ are the wire radius and the defect radius, respectively, $\left(x_{\mathrm{D}}, y_{\mathrm{D}}\right)$ is the location at the centre of the defect, and $\left(x_{1}, x_{\mathrm{s}}\right)$ are the limits of the defect along the $x$ axis. The defect parameters to be considered are angular location of defect $\left(\gamma_{\mathrm{D}}\right)$, width of the defect ( $w$ ), and height of defect $(t)$ (see Fig. 1b).

\section{Comparison between optical techniques and confocal microscopy}

In order to obtain the intensity distribution of the light beam scattered by the wire, it is necessary to know its topography $f(x)$. This topography has been

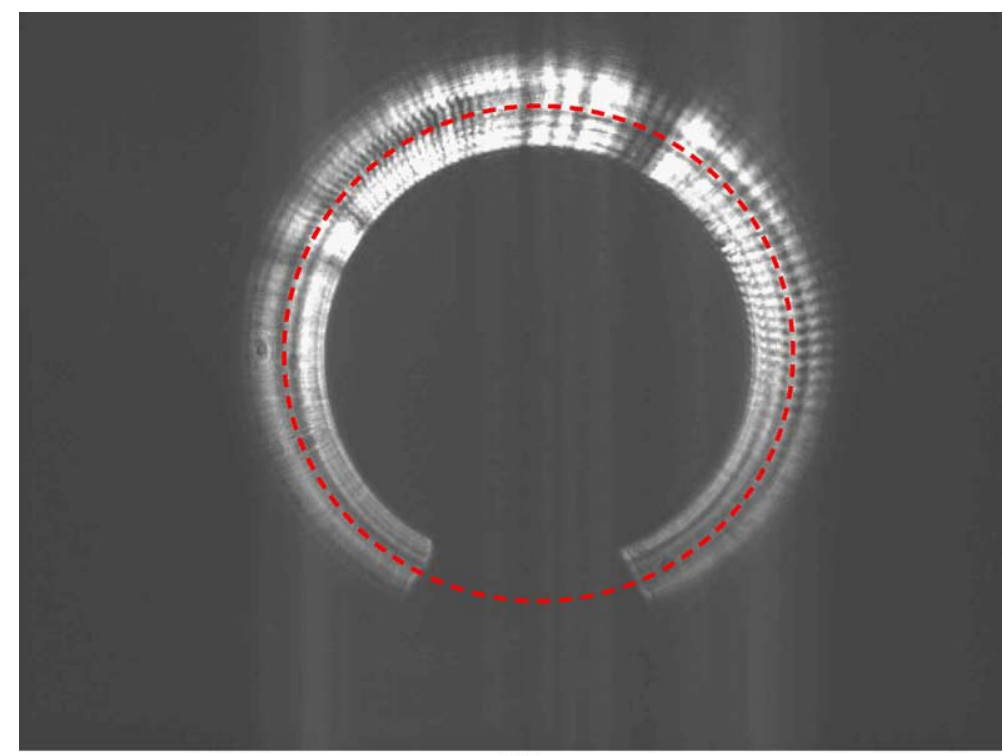

(a)

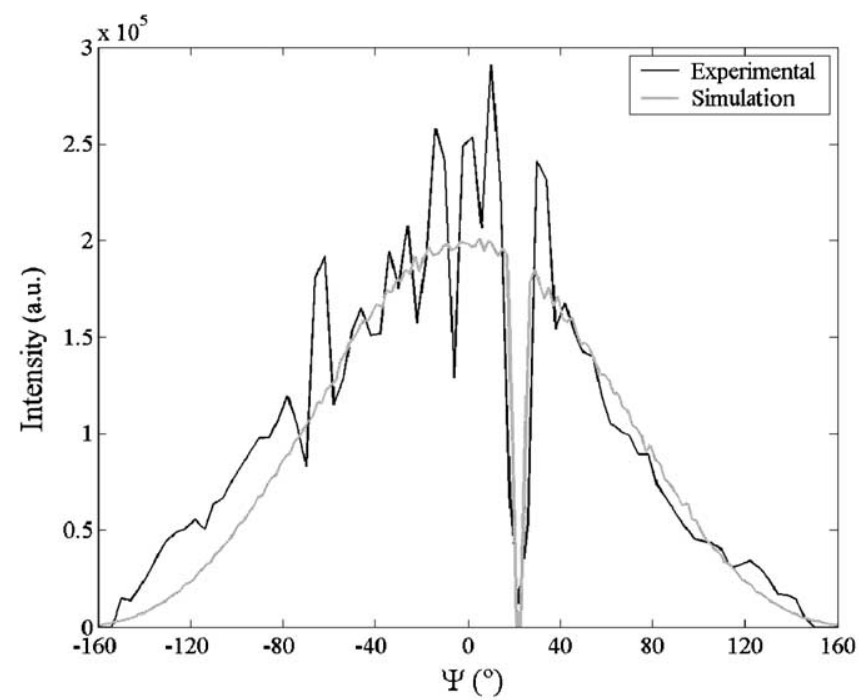

(b)

Fig. 3. (a) Experimental image for wire described in Fig. 2 when it is illuminated with a laser beam $(\lambda=632.8 \mathrm{~nm})$ in oblique incidences (angle between laser beam and wire axis $45^{\circ}$ ). The set-up is described in Sanchez-Brea et al. [4]. Normal incidence, described in this paper, is a particular case of this general set-up, and results are equivalent. (b) Comparison between experimental (black) intensity obtained in Fig. 3a (dashed circle), and model proposed in Eq. (5) when the topography is that of Fig. 2 (gray). Both intensity distributions are quite similar. 
obtained experimentally by using CM. In Fig. 2a, we can see an image and a transversal profile of a steel wire (wire radius $a_{0}=200 \mu \mathrm{m}$ ) with a dieline.

The profile of Fig. 2b has been fit to Eq. (6), thus considering the defect as a semicylinder. The dimensional parameters of the defect obtained with the fit are $a_{\mathrm{D}}=8 \mu \mathrm{m}, w=10 \mu \mathrm{m}(t=1.67 \mu \mathrm{m})$.

In Fig. 3a, we have also obtained an image when the wire is illuminated with a laser beam $(\lambda=632.8 \mathrm{~nm})$ in oblique incidence (angle between laser beam and wire axis $45^{\circ}$ ). The set-up is described in SanchezBrea et al. [4]. For our analysis we have used normal incident beam, but this is a particular case of the general set-up, and results are equivalent.

In Fig. 3b, we have compared the experimental intensity profile obtained for one ring (dash circle) that represents the different angular locations $\psi$, with the results obtained with Eq. (6). As it is shown, both intensity distributions are quite similar. Experimental intensity distribution presents more fluctuations. This is due to the presence of other defects on the surface, which have not been captured with the CM image.

\section{Conclusion}

A series of thin metallic wires with surface defects have been measured using confocal microscopy. Such topographical measurements have been used to validate an optical technique of surface characterization. This technique has been shown valid to detect on-line micrometric defects. The results obtained by the optical method, when the wire topography is considered, are in agreement with experiment. It is remarkable that the image processing required is not very time consuming and therefore defects can be detected in real time.

\section{Acknowledgements}

The authors thank Javier Alda for his valuable suggestions. This article is financed with DPI20011238 project.

\section{References}

[1] E. Bernabeu et al. Surface structures on fine and ultra-fine wires, Editorial Complutense. ISBN: 84-7491-679-8. Madrid 2002.

[2] E. Bernabeu, et al., Classification of surface structures on fine and ultra-fine wires, Appl. Surf. Sci. 180 (2001) 191-199.

[3] S. Gomez, K. Hale, J. Burrows, B. Griffiths, Measurements of surface defects on optical components, Meas. Sci. Technol. 9 (1998) 607-616.

[4] L.M. Sanchez-Brea, J.A. Gomez-Pedrero, E. Bernabeu, Measurement of surface defects on thin steel wires by atomic force microscopy, Appl. Surf. Sci. 150 (14) (1999) 125-130.

[5] L.M. Sanchez-Brea, et al., Optical technique for the automatic detection and measurement of surface defects on thin metallic wires, Appl. Opt. 39 (4) (2000) 539-545.

[6] L.M. Sanchez-Brea, E. Bernabeu, Diffraction by cylinders illuminated in oblique, off-axis incidence, Optik 112 (4) (2001) 169-174.

[7] J.C. Martínez Antón, P. Siegmann, L.M. Sánchez Brea, E. Bernabeu, In-line detection and evaluation of surface defects on thin metallic wires SPIE, Opt. Meas. Syst. Ind. Inspec. II: App. Prod. Eng. 4399 (2001) 27-31.

[8] M. Born, E. Wolf, Principles of Optics, sixth ed., Pergamon Press, Oxford, 1991. 